\title{
Numerical investigation on the thermal protection mechanism for blunt body with forward-facing cavity
}

\author{
LI XinDong, HU ZongMin* \& JIANG ZongLin \\ State Key Laboratory of High Temperature Gas Dynamics, Institute of Mechanics, Chinese Academy of Sciences, Beijing 100190, China
}

Received August 13, 2015; accepted December 21, 2015; published online January 29, 2016

\begin{abstract}
Numerical experiments are carried out using the standard hypersonic ballistic-type model (HB-2) to investigate the effect of forward-facing cavity on the aerodynamic heating. A general concept is proposed which utilizes the flow disturbances generated passively in the nosed subsonic region to weaken the detached shock wave. Several aspects are mainly studied, including shock shape and standoff distance, surface heat flux and pressure, flowfield feature and cooling mechanism. The numerical results indicate that shock strength and standoff distance increase with an increase in the $L / D$ ratio of the cavity. Interestingly, a bulge structure of the detached shock associated with a deep cavity is observed for the first time. Local surface heat flux and pressure around the concave nose are much lower respectively than those at the stagnation point of the baseline model. In addition, both surface heat and pressure reductions are proportional to the $L / D$ ratio. A negative heating phenomenon may occur in the vicinity of a sharp lip or on the base wall of a deep cavity. If the $L / D$ ratio exceeds 0.7 , the detached shock appears as a self-sustained oscillation which can be referred to as the cooling mechanism.
\end{abstract}

hypersonic flow, aerodynamic heating, thermal protection system, forward-facing cavity

Citation: $\quad$ Li X D, Hu Z M, Jiang Z L. Numerical investigation on the thermal protection mechanism for blunt body with forward-facing cavity. Sci China Tech Sci, 2016, 59: 1120-1129, doi: 10.1007/s11431-016-6015-4

\section{Introduction}

Hypervelocity vehicles, such as hypersonic aircrafts, high kinetic energy projectiles and missiles, suffer severe aerodynamic heating and drag. Such critical phenomena are associated with the formation of strong shock waves at the vehicle noses. For example, the stagnation temperature at the nose of a projectile flying at sea level and $2.6 \mathrm{~km} / \mathrm{s}$ corresponds to the melting point of tungsten $[1,2]$. The severe heating at the nose of a vehicle can change its shape due to surface melting and ablation, leading to unacceptable perturbations in aerodynamics and deviations in the flight trajectory. Therefore, it is of great importance to develop innovative active or passive techniques to reduce aerodynam-

*Corresponding author (email: huzm@imech.ac.cn) ic heating. Various techniques have been reported in literature, e.g., the spike ahead of a blunt body [3], concentrated energy deposition along the stagnation streamline [4], opposing jets [5], and combining jets with a forward-facing cavity in the stagnation zone of a blunt body [6]. However, system complexity is one of critical disadvantages of the preceding techniques.

Among all the aforementioned techniques, one passive design with a forward-facing cavity appears to be the simplest approach to reduce aerodynamic heating. The concept introduced by Hartmann and Troll [7] in 1922 involves the supersonic flow over a body with a forward-facing cavity for producing sound of high intensity and discrete frequencies, which is known as the "Hartmann Whistle". Since the late 1950s, a number of researchers have reported research efforts related to the idea of cavity flows. In 1958, the flight 
tests done by Hopko and Strauss [8] on a blunted cone with concave nose shape indicated that the heating at the stagnation point was reduced to one-tenth of that of convex noses at Mach 8.0. Burbank and Stallings [9] also observed a significant reduction in the stagnation-point heat transfer rate for concave hemispherical noses at supersonic Mach numbers, as compared with convex nose shapes. Johnson [10] found a very violent instability connected with a surface cavity in hypersonic helium flow over a blunt body. He pointed out that the instability mechanism is a vortex formation and shedding. Sambamurthi et al. [11] found the heat flux is less significant at the cavity base than it is at the nose rim. Later, they concluded that the shock oscillation frequency and amplitude are directly dependent on the cavity depth [12]. Yuceil et al. [1,2] indicated that large diameter shallow cavity created a stable "cool ring" in the vicinity of a sharp cavity lip, while rounding the cavity lip caused the temperature to rise to the level of the baseline case. However, Engblom and Goldstien [13] reported that rounding the cavity lip is necessary to reduce local surface heating due to airflow impinging on the cavity lip. In addition, they investigated the oscillations of hypersonic forward-facing cavity flow by experiments and numerical simulations [14-16]. Recently, Silton and Goldstein [17,18] used an axial cavity at the nose of a hypersonic vehicle to reduce severe heating and to delay the ablation onset in unsteady hypersonic flow. Saravanan et al. [19] observed experimentally and numerically a $35 \%-40 \%$ reduction in surface heating and 5\%-10\% reduction in drag for a missile shaped body at Mach 8.0, with a cavity located in the stagnation region at zero angle of incidence. Yadav and Guven [20] showed numerically that the heat transfer rate of a hemisphere-cylinder blunt body with a forward-facing parabolic cavity favorably reduced, and the same with a forward- facing ellipsoid cavity [21]. However, the local heating at the lip of the ellipsoid cavity became drastically high.

The main objective of the present study is to investigate the complex cavity flow for the thermal protection mechanics by the standard hypersonic ballistic model HB-2, which differs from other models in the previous literature. Some phenomena on the shape of detached shock wave and heat flux are presented for the first time. This paper is organized as follows. In section 2, we put forward a general concept for aerodynamic heating reduction, using a passive technique of forward-facing cavity as an example. Section 3 presents a detailed description of the numerical methods and mesh dependence study. The geometric configurations of HB-2 with concave noses for heat reduction is described particularly. Several aspects of the hypersonic cavity flows are investigated numerically in section 4, including the shock wave shape, the heat flux and pressure along the body surface, the unsteady flow features, and the cooling mechanism around forward-facing cavities. Conclusions are drawn in section 5 .

\section{The concept of heat reduction on blunt bodies}

Aerodynamic heating transferred from the gas into the body becomes a major consideration for the configuration design of a practical hypersonic vehicle. Theoretically, heat transfer rate is inversely proportional to the square root of the nose radius [22]. Therefore, aerodynamic heating at the nose can be considerably reduced for a blunt body as compared to a sharp-nosed body. This is why the hypersonic vehicles such as the Apollo space capsule and the space shuttle have blunt noses. As shown in Figure 1(a), there is a strong bow shock wave in front of the blunt nose, detached from the nose by a shock standoff distance $\delta$ [23]. Gases in the region between the bow shock and the body are very hot due to shock compression. Shock-wave heating and intense viscous dissipation are the causes of aerodynamic heating in the hypersonic flow over a blunt body.

The hot flowfield behind the bow shock can be divided into subsonic and supersonic regions by the sonic line, as sketched in Figure 1(a). Theoretically, the flow information may propagate everywhere in the subsonic or elliptic region while the flow information only propagates downstream along the characteristic lines in the supersonic or hyperbolic region. A natural concept is that the flow disturbances, if
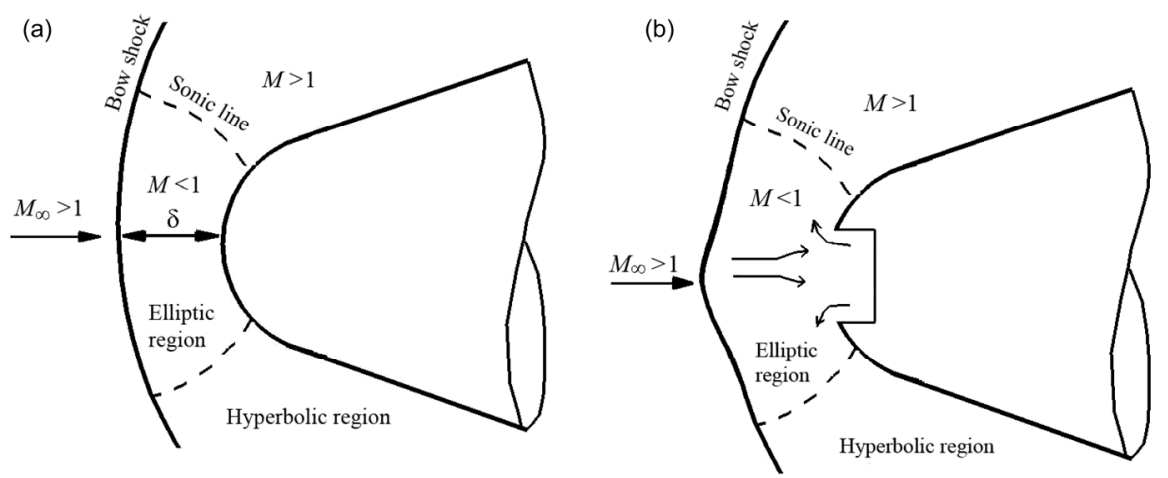

Figure 1 Schematic diagram of the flowfield over a hypersonic blunt-nosed body. (a) Without a cavity; (b) with a forward-facing cavity. 
they are generated passively in the subsonic region behind the strong bow shock, can propagate upstream in the elliptic region to weaken the shock wave. Due to these reversely propagating flow disturbances, the bow shock is pushed away from the nose, leading to an increase in the shock standoff distance; so much so that the bow shock shape may be changed eventually, as shown in Figure 1(b). This causes the aerodynamic heating to be reduced. In this paper, the cavity is studied as one of such passive techniques for thermal protection.

\section{Numerical methodology}

\subsection{Model configuration and governing equations}

The geometric configuration of the hypersonic ballistic-type model HB-2 [24], is presented in Figure 2 as a basic model for calculation. The baseline model is typically a blunted cone-cylinder-frustum with flared configuration, a $25^{\circ}$ cone half-angle and a nose radius of $0.3 d$, where $d$ is $0.1 \mathrm{~m}$. To reduce the computational cost, the viscous hypersonic flows are assumed to be axisymmetric using Navier-Stokes equations by finite difference method. The governing equations can be written in dimensionless conservation form as given in eq. (1):

$$
\frac{\partial \boldsymbol{U}}{\partial t}+\frac{\partial \boldsymbol{E}}{\partial x}+\frac{\partial \boldsymbol{F}}{\partial y}+\frac{1}{y} \boldsymbol{H}=\frac{1}{R e}\left(\frac{\partial \boldsymbol{E}_{v}}{\partial x}+\frac{\partial \boldsymbol{F}_{v}}{\partial y}+\frac{1}{y} \boldsymbol{H}_{v}\right),
$$

where $\boldsymbol{U}$ is the unknown state vector, $\boldsymbol{E}$ and $\boldsymbol{E}_{v}$ are the inviscid and viscous flux vectors along $x$-axis direction respectively, $\boldsymbol{F}$ and $\boldsymbol{F}_{v}$ are the inviscid and viscous flux vectors along $y$-axis direction respectively, and $\boldsymbol{H}$ and $\boldsymbol{H}_{v}$ are the source vectors. The details of the column vectors are given as follows:

$$
\begin{aligned}
\boldsymbol{U} & =\left(\begin{array}{l}
\rho \\
\rho u \\
\rho v \\
\rho E
\end{array}\right), \boldsymbol{E}=\left(\begin{array}{l}
\rho u \\
\rho u^{2}+p \\
\rho v u \\
(\rho E+p) u
\end{array}\right), \boldsymbol{F}=\left(\begin{array}{l}
\rho v \\
\rho u v \\
\rho v^{2}+p \\
(\rho E+p) v
\end{array}\right), \\
\boldsymbol{H} & =\left(\begin{array}{l}
\rho v \\
\rho u v \\
\rho v^{2} \\
(\rho E+p) v
\end{array}\right), \boldsymbol{E}_{v}=\left(\begin{array}{l}
0 \\
\tau_{x x} \\
\tau_{x y} \\
u \tau_{x x}+v \tau_{x y}-q_{x}
\end{array}\right)
\end{aligned}
$$

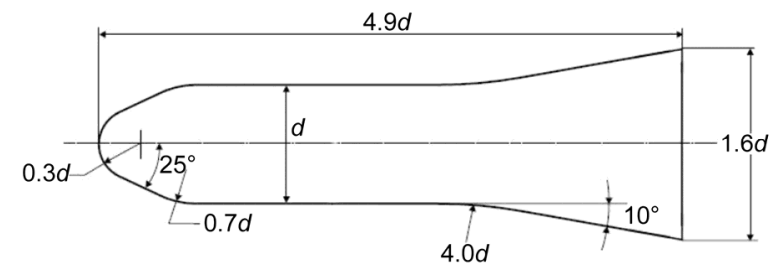

Figure 2 HB-2 standard model correlation configuration.

$$
\boldsymbol{F}_{v}=\left(\begin{array}{l}
0 \\
\tau_{x y} \\
\tau_{y y} \\
u \tau_{x y}+v \tau_{y y}-q_{y}
\end{array}\right),
$$

$\boldsymbol{H}_{v}=$

$\left(\begin{array}{l}0 \\ \tau_{x y}-\frac{2}{3} y \frac{\partial}{\partial x}\left(\frac{\mu v}{y}\right) \\ \tau_{y y}-\tau_{\theta \theta}-\frac{2}{3} \frac{\mu v}{y}-\frac{2}{3} y \frac{\partial}{\partial y}\left(\frac{\mu v}{y}\right) \\ u \tau_{x y}+v \tau_{y y}-\frac{2}{3} \frac{\mu v^{2}}{y}-\frac{2}{3} y \frac{\partial}{\partial y}\left(\frac{\mu v^{2}}{y}\right)-\frac{2}{3} y \frac{\partial}{\partial x}\left(\frac{\mu u v}{y}\right)-q_{y}\end{array}\right)$

The shear stress, heat flux and the state equation required to close the above system of equations are given by eqs. (3)-(5), namely

$$
\begin{gathered}
\tau_{x x}=\frac{2}{3} \mu\left(2 \frac{\partial u}{\partial x}-\frac{\partial v}{\partial y}\right), \tau_{y y}=\frac{2}{3} \mu\left(2 \frac{\partial v}{\partial y}-\frac{\partial u}{\partial x}\right), \\
\tau_{\theta \theta}=\frac{2}{3} \mu\left(2 \frac{v}{y}-\frac{\partial u}{\partial x}-\frac{\partial v}{\partial y}\right), \tau_{x y}=\mu\left(2 \frac{\partial u}{\partial y}+\frac{\partial v}{\partial x}\right), \\
q_{x}=-k \frac{\partial T}{\partial x}, q_{y}=-k \frac{\partial T}{\partial y}, \\
\rho E=\frac{p}{\gamma-1}+\rho \frac{u^{2}+v^{2}}{2}, p=\rho R T .
\end{gathered}
$$

The coefficient of shear viscosity $\mu$ in eq. (3) is computed by the Sutherland formula, while the thermal conductivity coefficient $k$ is derived from the Prandtl number.

The temporal discretization is obtained by a point implicit method and the spatial discretization is done using the second-order TVD scheme. The inviscid fluxes are computed using the HLLC Riemann solver [25] and the viscous terms are discretized with second-order central differencing. For the previous assumption of axisymmetric flows around model HB-2, an axis boundary condition implemented on the longitudinal axis in this problem holds

$$
v=0, \frac{\partial \rho}{\partial y}=\frac{\partial p}{\partial y}=\frac{\partial u}{\partial y}=0 .
$$

The surface of the baseline model is modeled as an isothermal wall at a temperature of $288.0 \mathrm{~K}$ with no slip velocity conditions, namely $u=0, v=0$. The freestream conditions [26] are given in Table 1, which are imposed on the inlet and the initialization for all grids. All calculations are done by several simplifying assumptions, such as the axisymmetric flow, the calorically perfect gas and the laminar 
Table 1 Free stream conditions

\begin{tabular}{ccc}
\hline Symbol & Quantity & Value \\
\hline$M a_{\infty}$ & Mach number & 9.7 \\
$R e_{\infty}$ & Reynolds number & $4.0 \times 10^{6}$ \\
$P_{\infty}(\mathrm{Pa})$ & Pressure & 168.1 \\
$u_{\infty}(\mathrm{m} / \mathrm{s})$ & Velocity & 1464 \\
$T_{\infty}(\mathrm{K})$ & Free stream temperature & 56.8 \\
$T_{\mathrm{w}}(\mathrm{K})$ & Wall temperature & 288.0 \\
\hline
\end{tabular}

flow. The actual Reynolds number is much smaller along the surface inside and outside the cavity due to the low speed flow, so transition does not occur within the regions of interest in this study.

\subsection{Grid independence study for the baseline model}

The grid independence study is conducted via calculation of the baseline model HB-2 without a concave nose. Four groups of coarse and fine grids are given in Table 2 for verification. It shows that the stagnation point values of heat flux and pressure only change $3.2 \%$ and $0.06 \%$ respectively when the mesh size goes from $250 \times 600$ to $300 \times 800$. In Figure 3(a), the heat flux along the surface of the baseline model without cavity demonstrates that the finest mesh is sufficient to resolve the stagnation heating. As can be seen from Figure 3(b), the surface pressure profiles closely coincide with each other for all grids. Therefore, the finest grids of $300 \times 800$ can be used to compute the cavity flows in the next section.

\subsection{Grid generation for the models with cavity}

All the geometries with a cavity at the nose of HB-2 used in the study are discretized into multi-block, structured grids, as shown in Figure 4. Inside the cavity, the base wall is defined as the surface region from points $\mathrm{A}$ to $\mathrm{B}$, and the region from points $B$ to $C$ is called the side wall. The surface along the body of HB-2 between the points $\mathrm{C}$ and $\mathrm{F}$ is the outer solid wall of the cavity. Outflow, farfield and axisymmetric boundary conditions are imposed on surfaces FG, GH and AH, respectively. The cavity considered in the evaluation of model configuration is called a "shallow" or "deep" cavity according to its length $(L)$; correspondingly a "small" or "large" cavity is named according to its diameter (D), as sketched in Figure 4.

In Table 3 , the diameter $(D)$ of large and small cavities are $0.3 d$ and $0.2 d$ respectively for the calculation, where $d=$ $0.1 \mathrm{~m}$. Various length-to-diameter ratios are investigated for the hypersonic flow at Mach 9.7, namely $L / D=0.2,0.5,0.7$,

Table 2 Grid independence study

\begin{tabular}{|c|c|c|c|c|}
\hline Group & Grids & The first layer mesh (m) & Stagnation heat flux $\left(\mathrm{kW} / \mathrm{m}^{2}\right)$ & Stagnation pressure $(\mathrm{kPa})$ \\
\hline 1 & $100 \times 300$ & $10^{-5}$ & 491.470 & 20.156 \\
\hline 2 & $200 \times 450$ & $10^{-6}$ & 350.176 & 20.179 \\
\hline 3 & $250 \times 600$ & $10^{-7}$ & 327.436 & 20.194 \\
\hline 4 & $300 \times 800$ & $10^{-7}$ & 317.158 & 20.207 \\
\hline
\end{tabular}
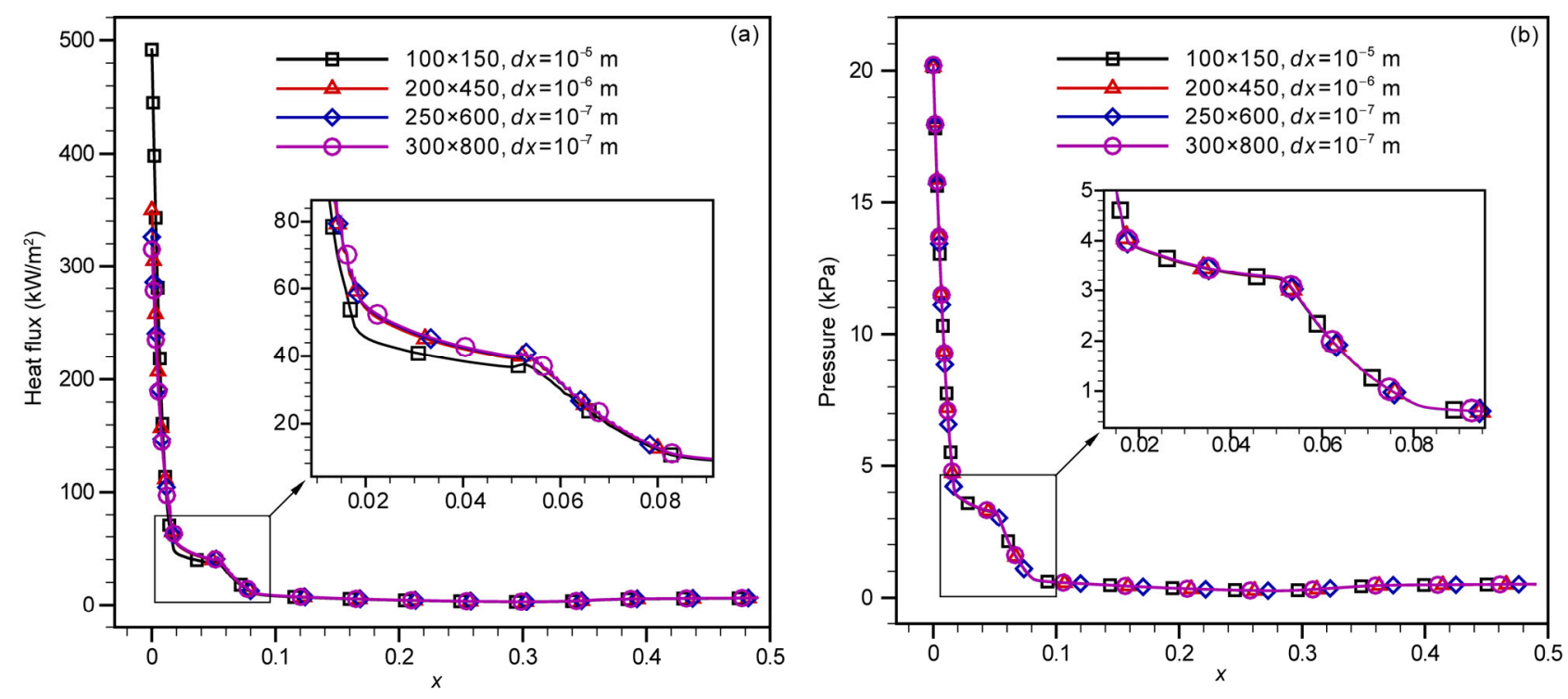

Figure 3 (Color online) Surface heat flux and pressure distribution along the baseline model without a cavity for coarse and fine grids. (a) Surface heat flux; (b) surface pressure. 


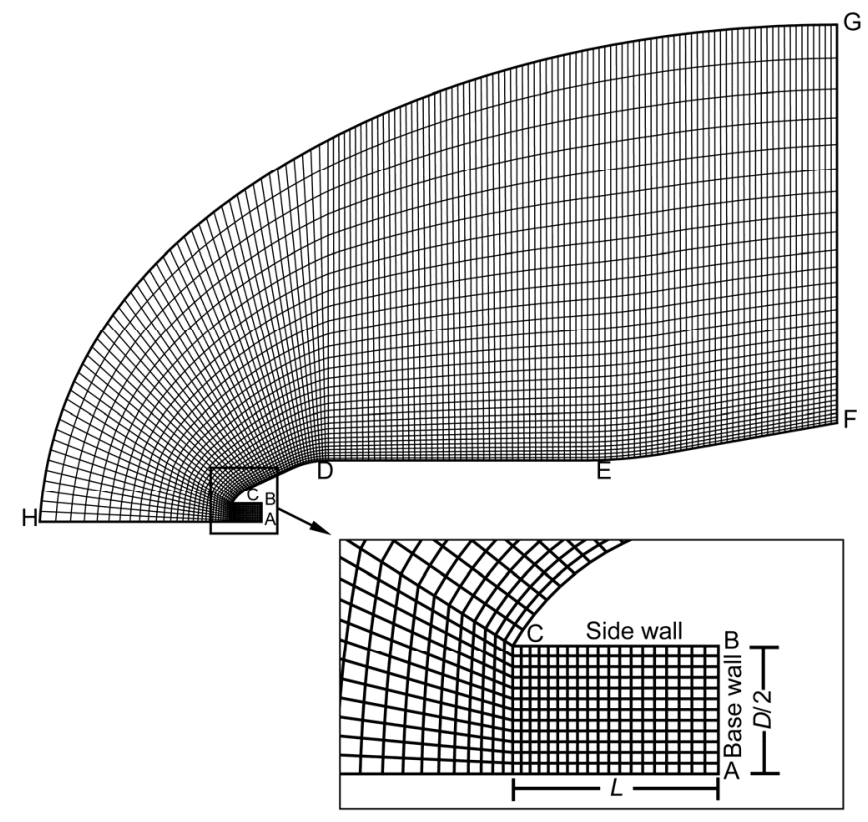

Figure 4 Schematic diagram of the cavity configuration and grids $(L / D$ $=1.6$ ).

1.0, 1.3, 1.6 and 2.0. The computational domain is discretized into $300 \times 800$ mesh nodes in accordance with the grid independence study in section 3.2. Inside the cavity, there
Table 3 Cavity sizes used for calculation

\begin{tabular}{ccccccc}
\hline \multirow{2}{*}{ Cases } & \multirow{2}{*}{$L / D$} & \multicolumn{2}{c}{ Small cavities } & & \multicolumn{2}{c}{ Large cavities } \\
\cline { 3 - 4 } \cline { 5 - 6 } & & $L(\mathrm{~m})$ & $D(\mathrm{~m})$ & & $L(\mathrm{~m})$ & $D(\mathrm{~m})$ \\
\hline 1 & 0.2 & 0.004 & 0.02 & & 0.006 & 0.03 \\
2 & 0.5 & 0.010 & 0.02 & & 0.015 & 0.03 \\
3 & 0.7 & 0.014 & 0.02 & & 0.021 & 0.03 \\
4 & 1.0 & 0.020 & 0.02 & & 0.030 & 0.03 \\
5 & 1.3 & 0.026 & 0.02 & & 0.039 & 0.03 \\
6 & 1.6 & 0.032 & 0.02 & & 0.048 & 0.03 \\
7 & 2.0 & 0.040 & 0.02 & & 0.060 & 0.03 \\
\hline
\end{tabular}

are 151 grid points along the base wall for all cavities. Different grid points along the side wall correspond to different cavity lengths, e.g., 91 grid points for $L / D=0.2$ while 251 for $L / D=2.0$.

\section{Results and discussion}

\subsection{Shock wave shape and shock standoff distance}

The numerical schlieren picture of the detached shock wave around the baseline model without a cavity, as shown in Figure 5(a), consists of a stationary bow shock wave in front of the blunt body. However, the shock wave shape for

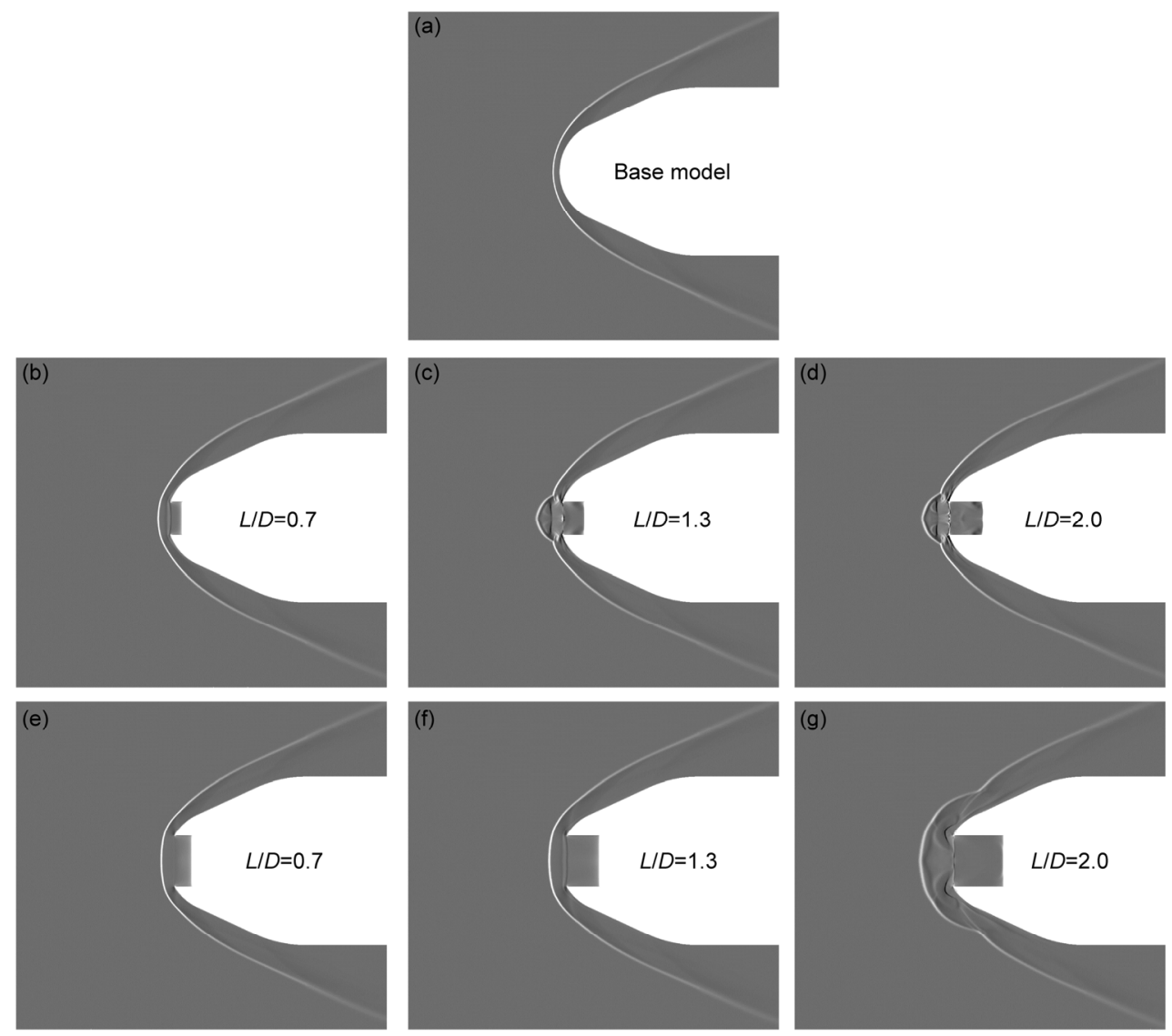

Figure 5 Numerical schlieren pictures of the detached shock wave. (a) the baseline model; the small cavities ( $D=0.02 \mathrm{~m})$ : (b) $L / D=0.7$, (c) $L / D=1.3$, (d) $L / D=2.0$; the large cavities ( $D=0.03 \mathrm{~m}$ ): (e) $L / D=0.7$, (f) $L / D=1.3$, (g) $L / D=2.0$. 
cavity flows shows a big difference from that of the baseline model. Figure 5(b)-(g) depicts the flow structures respectively for the small and large cavities, each of which includes three length-to-diameter ratios, namely $L / D=0.7,1.3$ and 2.0. Two significant phenomena can be obviously seen from these schlieren pictures. One of the phenomena is that the bow shock wave as shown in Figure 5(b), (e) and (f) presents a normal segment almost up to the cavity lip, with a larger shock standoff distance than that of the baseline model as given in Figure 5(a). Such a phenomenon has been mentioned in literature regarding the shallow cavities. A new phenomenon is observed in Figure 5(c), (d) and (g). The normal shock fragment turns into a bulge structure when $L / D=1.3$ or 2.0 for the small cavity, or when $L / D=2.0$ for the large cavity. The cause and transition mechanism of the bulge structure will be analyzed in section 4.4. There is a significant increase of the shock standoff distance due to the bulge structure, which may have an important impact on the surface heat flux and pressure distribution.

\subsection{Effect of cavity on the surface heat flux}

The surface heat flux for the hypersonic ballistic-type model HB-2 with various cavity configurations are shown in Figure 6 . The heat flux along the body surface inside and outside the small cavities $(D=0.02 \mathrm{~m})$ in Figure 6(a)-(c) are normalized by the peak heating value of the baseline model without a cavity. The surface heating profiles for the large cavities $(D=0.03 \mathrm{~m})$ are also shown in Figure 6(d)-(f). Three regions of the body surface, e.g., the base wall, the
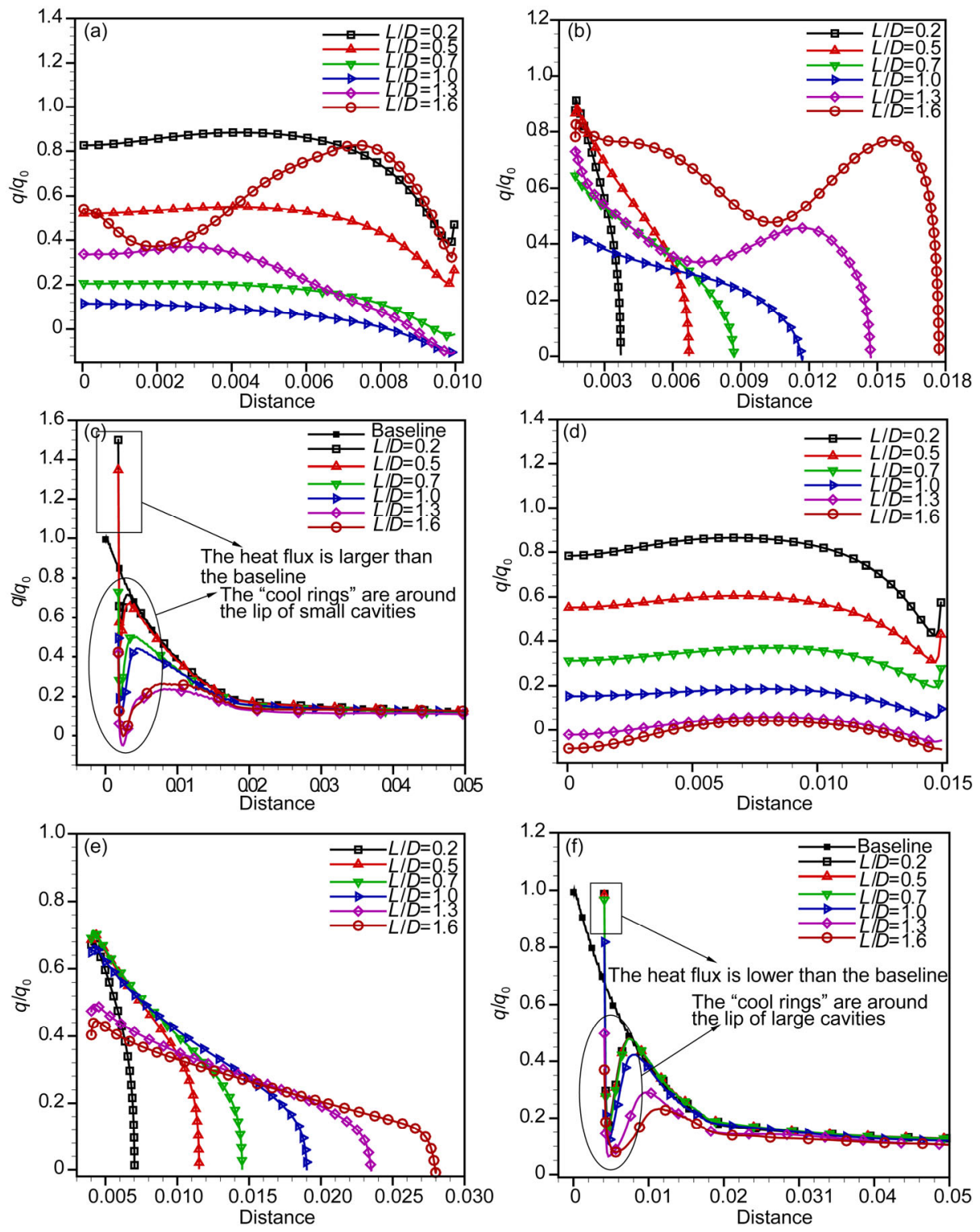

Figure 6 (Color online) Normalized heat flux along the body surface inside and outside of the cavities with different $L / D$ ratios. Small cavity $(D=0.02 \mathrm{~m})$ : (a) base wall; (b) side wall; (c) outer surface of the cavity. Large cavity ( $D=0.03 \mathrm{~m}$ ): (d) base wall; (e) side wall; (f) outer surface of the cavity. 
side wall and the outer surface of the cavity lip as sketched in Figure 4, are investigated carefully with $L / D=0.2,0.5,0.7$, $1.0,1.3$ and 1.6 for different computations. With the detached shock appears unsteadiness, all the heat flux profiles in Figure 6 correspond to the transience when each shock wave reaches the largest shock standoff location.

The normalized heat transfer rates on the base walls of the small and large cavities are all below unity in Figure 6(a) and (d), indicating a significant reduction as compared with the stagnation point value of the baseline model. The maximum reduction occurs when $L / D=1.0$ for the small cavity as shown in Figure 6(a), while it occurs when $L / D=1.6$ for the large cavity as shown in Figure 6(d). However, for the small cavities in Figure 6(a), the heat transfer rates at the cavity corner (i.e. point B in Figure 4) are surprisingly negative for $L / D=1.0$ and 1.3 , marked by a right triangle and a diamond, respectively. For the large cavities in Figure 6(d), the surface heat flux are almost totally below zero if $L / D=1.3$ and 1.6 marked by a diamond and a circle, respectively. The heating reduction on the base walls of large cavities is proportional to the length of the cavity.

As depicted in Figure 6(b) and (e), the normalized heat flux in both small and large cavities are decreased to be below unity on the side wall (the surface BC in Figure 4). For the large cavities in Figure 6(e) where $D=0.03 \mathrm{~m}$, the heat transfer rate near the cavity lip reduces sharply with an increase in the cavity length. As shown in Figure 6(b) for the small cavities, the maximum reduction of the heat flux near the cavity lip occurs when $L / D=1.0$.

The aerodynamic heating along the outer surface of the cavity is compared with the baseline configuration in Figure 6(c) and (f). The profile of the baseline model is marked by the closed square. In the vicinity of the sharp lip, the heat flux for each case with a cavity is lower than the stagnation point value of the baseline model, except for the small cavities when $L / D=0.2$ and 0.5 , as respectively labeled by an open square and a triangle in Figure 6(c). For most of the studied length-to-diameter ratios, the "cool rings" [1,2] on the outer surface of the sharp lip appear in the cavity flows, as encircled by an oval in Figure 6(c) and (f), respectively. Furthermore, the minimum heat flux among the cool rings also shows a negative value when $L / D=1.3$ in the small cavity, as shown in Figure 6(c). For the large diameter cavities in Figure 6(f), the heating reduction is also in proportion to the cavity depth. The larger the $L / D$, the better the cooling effect of the cavity.

During the detached shock movement, the singular point $\mathrm{C}$ at the sharp lip in Figure 4 will suffer severe heating. The normalized value of minimum/maximum heat flux at point $\mathrm{C}$ is $1.50 / 1.51,1.33 / 1.62,0.73 / 2.20,0.50 / 2.30,0.41 / 3.13$, and $0.42 / 3.57$, corresponding to the small cavities with $L / D$ $=0.2,0.5,0.7,1.0,1.3$, and 1.6, respectively. Similarly in the large cavities, the minimum/maximum heat flux is $0.987 / 0.998,0.982 / 0.993,0.97 / 1.09,0.82 / 1.38,0.50 / 2.02$, and $0.37 / 3.47$. The small cavities with $L / D=0.2$ and 0.5 have no heating reduction, while the large cavities with these two $L / D$ ratios have a heat drop all the time. Although there exists maximum heating increase in cavities with the ratio 0.7 to 1.6, the large deep cavities have a better cooling effect than small deep cavities.

\subsection{Effect of cavity on the surface pressure}

The surface pressure distributions investigated for all cavity configurations are found to follow the same tendency, so only the large cavities are discussed in this section, which are shown in Figure 7(a)-(d). Obviously, the pressure distributions remain almost constant along the base wall of the cavity in Figure 7(a). The pressure drop is directly proportional to the $L / D$ ratio of the cavity. A similar trend in the surface pressure on the side wall can be observed in Figure 7(b). The surface pressure close to the cavity lip is the lowest. In Figure 7(c), there is a significant pressure reduction at the outer surface of the lip, compared with the stagnation point pressure, as encircled by an oval. The larger the $L / D$, the larger the drop in the surface pressure around the concave nose. However, in Figure 7(d), there is no much effect on the surface pressure away from the nose region, especially for the outer surface of the afterbody.

\subsection{Flowfield features and flow unsteadiness}

The flowfield feature is significantly altered by the presence of a forward-facing cavity at the nose of HB-2, as can be seen in Figure 8(a)-(d), which correspond to different flow time. The flow time is defined as the ratio of cavity length to the average flow velocity inside the cavity. The evolution of streamlines surrounding a concave nose shows a cycle motion of unsteadiness, which is analyzed for the small cavity with $L / D=1.3$.

In Figure 8(a), the inflow enters into the cavity, directly impinging on the base wall where the surface pressure increases and some small vortexes are developed. With time proceeding, these small vortexes grow into several primary ones, leading to the pressure increasing continually. The unsteady pressure interaction inside the cavity generates a growing pocket of high pressure fluid, which begins to push the incoming flow away from the body, resulting in the formation of a singular point on the symmetry line. In Figure $8(\mathrm{c})$, at $t=4.7 \times 10^{-4}$ second, a pair of dominant recirculation zones gradually moves upstream. Simultaneously, the airflow is coming out of the cavity, and results in the formation of a symmetrical oblique shock due to a reverse collision between the inflow and the outflow. Consequently, the bow shock standing in front of the blunt body grows into a bulge structure until the growth of the oblique shock halts, as shown clearly in Figure 8(d). In addition, the outflow separates and forms a secondary recirculation zone just outside of the cavity lip within the primary vortex, which leads to the phenomenon of a cool ring as mentioned in sec- 

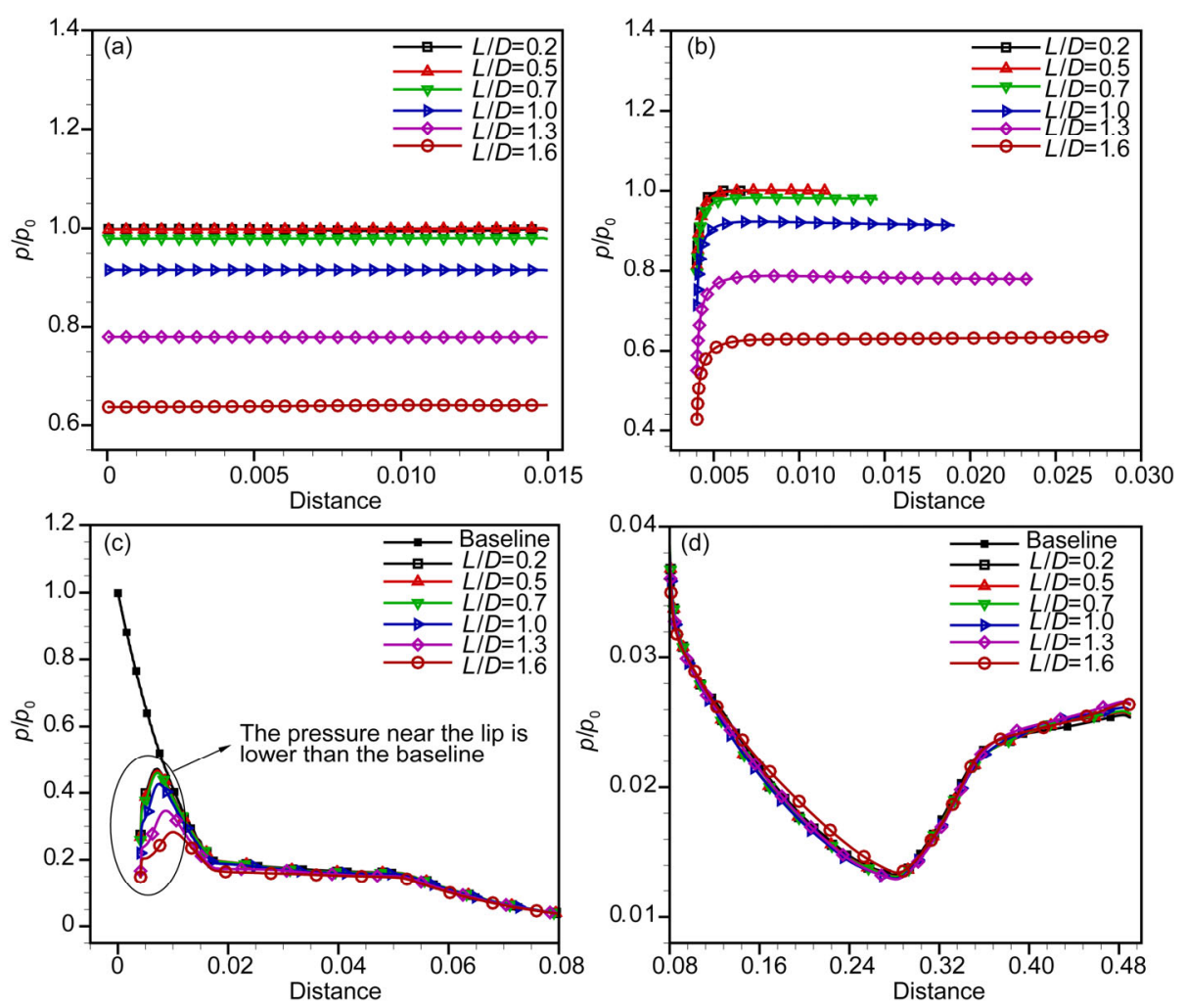

Figure 7 (Color online) Normalized pressure distribution along the body surface inside and outside of the large cavities with different $L / D$ ratios $(D=0.03$ $\mathrm{m}$ ). (a) Base wall; (b) side wall; (c) outer surface of the cavity; (d) outer surface of the afterbody.
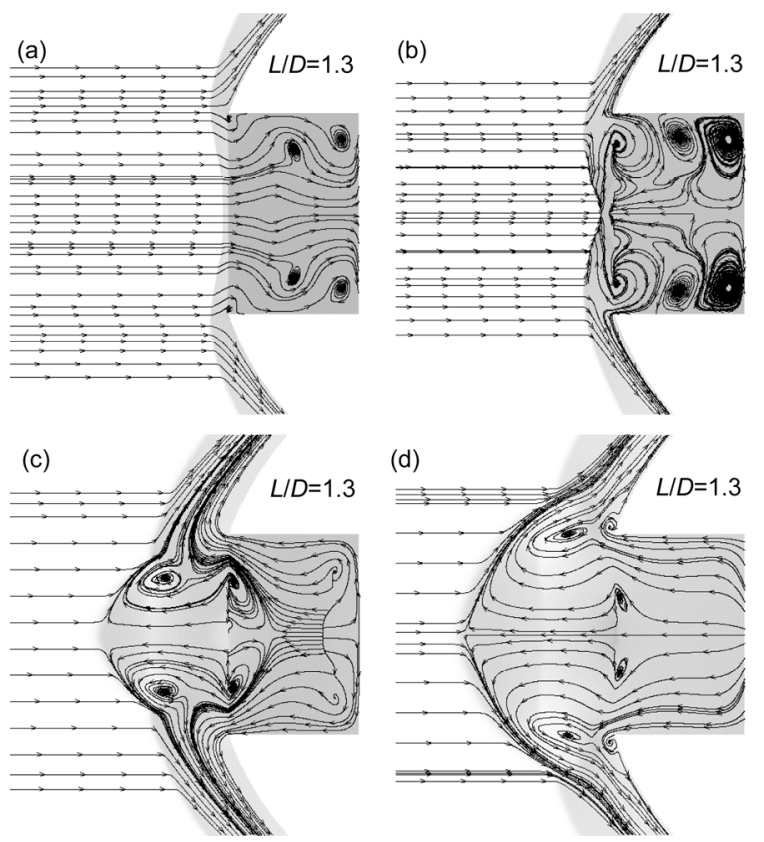

Figure 8 The streamlines around the small cavity with $L / D=1.3$ at different time. (a) $t=4.2 \times 10^{-4} \mathrm{~s}$; (b) $t=4.5 \times 10^{-4} \mathrm{~s}$; (c) $t=4.7 \times 10^{-4} \mathrm{~s}$; (d) $t=4.8 \times 10^{-4} \mathrm{~s}$.

tion 4.2 .

In Figure 9(a) and (b), respectively, the pressure oscillations are investigated on the base wall of the small and large cavities with various $L / D$ ratios. All the test positions are located at the middle point of the base wall, i.e. point $\mathrm{A}$ in Figure 4. For the small cavities in Figure 9(a), the deep cavity has larger amplitude of longitudinal pressure oscillation and lower oscillation frequency than that of the shallow cavity. The pressure oscillation appears as a periodic phenomenon in the deep cavities, except for when $L / D=0.2$. In Figure 9(b), the large cavities have similar results. For a fixed value of the $L / D$ ratio, the periodic time of pressure oscillation is shorter in the large cavities than it is in the small cavities. For all cavities in Figure 9(a) and (b), it is shown that the cycle of unsteady flows occurs most easily in the deep cavity. If the $L / D$ ratio of the cavity exceeds 0.7 , a self-sustained resonant oscillation flow exists, which is in accordance with the periodic movement of the detached shock wave.

\subsection{Cooling mechanism}

A forward-facing cavity flow at hypersonic Mach numbers has oscillating pressure levels, which is one of the dominant flow features. The heat flux calculated in this paper for cavity configurations are lower than that of similar flows wherein not enough oscillations are present. The experimental and numerical investigations showed a heat reduction in the vicinity of the cavity lip due to the presence of a cool ring $[2,13,15,19]$. 

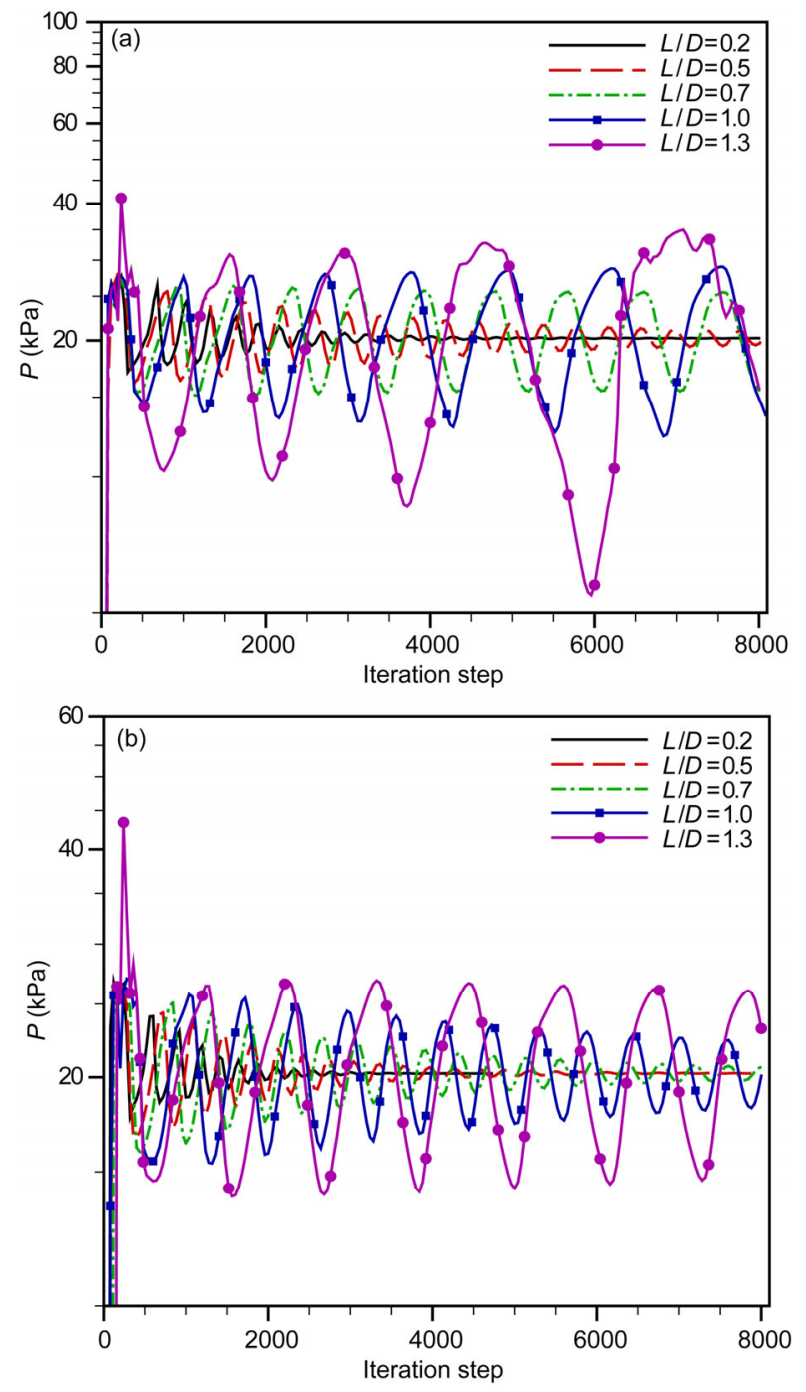

Figure 9 (Color online) The pressure oscillations on the base wall of the cavities with various $L / D$ ratios. (a) The small cavities; (b) the large cavities.

In Figure 8(a)-(d), near the middle point A (as shown in Figure 4) of the base wall, the Mach numbers respectively are $0.06,0.01,0.02$, and 0.05 , and the temperature are 1139 , 1120,978 , and $899 \mathrm{~K}$, respectively. In Figure 8(a), when the detached shock is moving toward the cavity mouth, the temperature of the inflow air near the base wall is a little higher than that of the baseline model at the stagnation position (1125 K). However, in Figure 8(c)-(d), when the detached shock is moving upstream, the hot air inside the cavity begins to flow out and the Mach number near the base wall increases gradually from 0.01 to 0.05 . Conversely, the temperature decreases from 1120 to $899 \mathrm{~K}$, which means the internal energy of fluid particles translate partly into kinetic energy. As a result, the whole flow field inside the cavity cools down. On the other hand, the outflows also form a cool separation recirculation zone around the sharp lip, which protects the outer surface from the convective heat transfer by the extremely hot air, as shown in Figure $8(\mathrm{~d})$.
Therefore, the cooling mechanism might be interpreted as the unsteady oscillation flows caused by the cavity propagate upstream in the subsonic region to push the detached shock away from the concave nose, leading to the acceleration of low temperature inside the cavity and the formation of a cool ring by the outflows outside the cavity. In addition, unsteady movement of the detached shock is necessary to produce reduction of the aerodynamic heating.

\section{Conclusions}

The standard hypersonic ballistic model HB-2 with a forward-facing cavity was investigated numerically as a passively thermal protection technique against aerodynamic heating. Two groups of unique cavities with different length -to-diameter ratios were simulated and discussed in detail. The findings are summarized as follows.

1) Surface heat flux around the concave nose had a significant reduction for all cavity configurations, except for small cavities in the $L / D$ ratios of 0.2 and 0.5 . For cavities with the same diameter, the heating drop and pressure drop were both directly proportional to the $L / D$ ratio, i.e. the deeper the cavity, the larger the reduction of heat flux and pressure. Especially for a very deep cavity, the negative heat flux may occur on the base wall or at the outer surface of cavity lip. For a fixed $L / D$, the ability to reduce heating in the large deep cavities was better than it was in the small deep cavities.

2) Forward-facing cavities had a common feature of pressure oscillation at hypersonic flows. The deeper the cavity, the higher the amplitude of oscillation pressure and the lower was the oscillation frequency. Surface nose-tip cooling was achieved by creating strong pressure oscillations, which led to the large standoff distance of the shock wave. The bulge structure of the detached shock in front of the concave nose was first observed in the present numerical study. This bulge appeared if the pressure oscillations were strong enough. Further, when the $L / D$ ratio of the cavity was more than 0.7 , the detached shock had a motion of self-sustained oscillation.

3) The cooling mechanism of the forward-facing cavity was mainly caused by the oscillation motions of the cavity flow, which produced a substantial heating reduction.

This work was supported by the National Natural Science Foundation of China (Grant No. 11532014).

1 Yuceil B, Dolling D S, Wilson D. A preliminary investigation of the helmholtz resonator concept for heat flux reduction. AIAA J, 1993, 2742

2 Yuceil, B, Dolling, D S. Effect of a nose cavity on heat transfer and flowfield over a blunt body at mach 5. AIAA J, 1994, 2050

3 Jiang Z L, Liu Y F, Han G L, et al. Experimental demonstration of a new concept of drag reduction and thermal protection for hypersonic vehicles. Acta Mech Sinica, 2009, 25: 417-419 
4 Satheesh K, Jagadeesh G. Effect of concentrated energy deposition on the aerodynamic drag of a blunt body in hypersonic flow. Phys Fluids, 2007, 19: 1-4

5 Hayashi K, Aso S, Tani Y. Numerical study on aerodynamic heating reduction by opposing jet. Memoirs Faculty Eng Kyushu Univ, 2006, 66: 39-44

6 Lu H B, Liu W Q. Research on thermal protection mechanism of forward-facing cavity and opposing jet combinatorial thermal protection system. Heat Mass Transfer, 2014, 50: 449-456

7 Hartmann J, Troll B. On a new method for the generation of sound waves. Phys Rev, 1922, 20: 719-727

8 Hopko R N, Strauss H K. Some Experimental heating data on convex and concave hemispherical nose shapes and hemispherical depressions on a 30 degree blunted nose cone. NACA RM-L58A17a, 1958

9 Burbank P B, Stalling R L. Heat transfer and pressure measurements on flat nose cylinder at a Mach number range of 2.49 to 4.44. NASA TM X-221, 1959

10 Johnson R H. Instability in hypersonic flow about blunt bodies. Phys Fluids, 1959, 2: 526-532

11 Sambamurthi J K, Huebner L D, Utreja L R. Hypersonic flow over a cone with nose cavity. AIAA Paper, 1987, 1193

12 Huebner L D, Utreja L R. Mach 10 bow-shock behavior of forward-facing nose cavity. J Spacecraft Rockets, 1993, 30: 291-297

13 Engblom W A, Goldstein D B. Nose-tip surface heat reduction mechanism. J Thermophys Heat Tr, 1996, 10: 598-606

14 Engblom W A, Yuceil B, Goldstein D B, et al. Hypersonic forward-facing cavity flow: An experimental and numerical study. AIAA J, 1995, 0293

15 Engblom W A, Yuceil B, Goldstein D B, et al. Experimental and numerical study of hypersonic forward-facing cavity flow. J Spacecraft Rockets, 1996, 33: 353-359

16 Engblom W A, Goldstein D B, Ladoon D, et al. Fluid dynamics of forward-facing cavity flow. J Spacecraft Rockets, 1997, 34: 437-445

17 Silton S I, Goldstein D B. Ablation onset in unsteady hypersonic flow about nose tip with cavity. J Thermophys Heat Tr, 2000, 14: 421-434

18 Silton S I, Goldstein D B. Use of an axial nose-tip cavity for delaying ablation onset in hypersonic flow. J Fluid Mech, 2005, 528: 297-321

19 Saravanan S, Jagadeesh G, Ressy K P J. Investigation of missile-shaped body with forward-facing cavity at Mach 8 . J Spacecraft Rockets, 2009, 46: 577-591

20 Yadav R, Guven U. Aerothermodynamics of a hypersonic vehicle with a forward-facing parabolic cavity at nose. J Aerospace Eng, 2013: 1-12

21 Yadav R, Guven U. Aerothermodynamics of a hypersonic projectile with forward-facing ellipsoid cavity at nose. J Spacecraft Rockets, 2015, 52: 157-165

22 Anderson J D. Hypersonic and High Temperature Gas Dynamics. 2nd ed. Virginia: AIAA, 2006

23 Anderson J D. Computational Fluid Dynamics: the Basics with ApPlications. New York: McGraw Hill, 1995

24 Gray J D, Lindsay E E. Force tests of standard hypervelocity ballistic model HB-1 and HB-2 at Mach 1.5 to 10. AEDC-TDR-63-137, 1963

25 Toro E F. Riemann Solvers and Numerical Methods for Fluid Dynamics. 3rd ed. Berlin: Springer, 2009

26 Shigeru K I, Shigeya W, Shinji N, et al. Comparative force/heat flux measurements between JAXA hypersonic test facilities using standard model HB-2 (Part 1: $1.27 \mathrm{~m}$ hypersonic wind tunnel results). JAXA Research and Development Report, 2005, ISSN 1349-1113 